\title{
Meningioma de la Vaina del Nervio Optico (MVNO). ¿La radioterapia es el actual patrón de cuidados? A propósito de un caso y revisión de la literatura
}

\author{
M. Caeiro ${ }^{1}$, C. Conde ${ }^{2}$, M. L. López ${ }^{1}$, L. Pérez ${ }^{1}$, M. L. Vázquez de la Torre ${ }^{1}$, M. Canteli ${ }^{1}$, A. Alonso ${ }^{1}$, D. Medal ${ }^{4}$, \\ M. Salgado ${ }^{4}$, J. Brasa ${ }^{3}$, J. M. Otero ${ }^{2}$, V. M. Muñoz ${ }^{1}$
}

\section{Resumen}

El meningioma primario de la vaina del nervio óptico es un tumor raro, cuyo manejo diagnóstico y terapéutico ha variado de manera sustancial en los últimos años. La aparición y desarrollo de las técnicas de diagnóstico por imagen, como son la Tomografía Computerizada de Alta Resolución y la Resonancia Magnética Nuclear, han permitido diagnósticos más precoces y precisos.

La introducción de la Radioterapia Convencional (RT) a finales de los años 70, sola o en combinación con la cirugía, pero sobre todo la introducción de la Radioterapia Extereotáxica Fraccionada (RTEF) y la Radioterapia Conformacional Tridimensional (RTC3D), ha supuesto una alternativa terapéutica sumamente eficaz y con una toxicidad relativamente baja.

En este artículo revisamos en primer lugar el desarrollo embriológico, anatomía, la historia natural, el tratamiento quirúrgico, así como el papel de la RT en esta entidad, y su integración en el patrón de cuidados actual. Analizamos las diferentes técnicas radioterápicas desde las más elementales como la Radioterapia Convencional (RT), siguiendo con la Radioterapia Conformada 3 D (RTC3D), hasta las más sofisticadas como la Radioterapia Exterotáxica Fraccionada (RTEF), todo ello a propósito de nuestra experiencia en un caso.

Palabras clave:

Meningioma. Nervio Óptico. Radioterapia Extereotáxica Fraccionada.

Oncología, 2006; 29 (1):30-38

\footnotetext{
${ }^{1}$ Oncología Radioterápica (Instituto Galego de Medicina Técnica)

${ }^{2}$ Neurocirugía

${ }^{3}$ Diagnóstico por Imagen (Instituto Galego de Medicina Técnica)

${ }^{4}$ Radiofísica Hospitalaria (Instituto Galego de Medicina Técnica)

Dependientes del Complejo Hospitalario Universitario de Vigo (CHUVI)
} 


\section{Summary}

Primary optic nerve sheat meningioma (ONSM) is a rare tumor, whose diagnosis and therapeutic management have varied in a substantial manner over the last few years. The appearance and development of diagnostic imaging techniques such as High Resolution Computerized Tomography and Nuclear Magnetic Resonance have allowed earlier and more precise diagnoses.

The introduction of conventional radiation therapy in the late 1970s, alone or in combination with surgery, and above all the introduction of Fractionated Stereotactic Radiotherapy and Three-Dimensional Conformal Intensity-Modulated Radiotherapy (3DCRT) have provided very efficient alternative therapies with a relatively low toxicity.

In this article we first review the embryological development, anatomy, natural history, and surgical treatment, and then describe the role of radiotherapy (RT) in the considered entity, indicating how it can be integrated in the pattern of present day care.

We analyse the different radiotherapy techniques, from the most elementary, such as conventional RT, followed by 3DCRT up to the most sophisticated such as Stereotactic Fractionated Radiotherapy, all based on our experience in one case.

Key words: Meningioma. Optic nerve. Fractionated Stereotactic Radiotherapy.

\section{Introducción}

El meningioma de la vaina del nervio óptico (MVNO) es una entidad clínico-patológica rara, que constituye alrededor del $1-2 \%$ de todos los meningiomas. Estos pueden originarse en las meninges del nervio óptico intraorbitario (meningioma de la vaina del nervio óptico primaria) o en las meninges del canal óptico o hueso esfenoidal. Hay otros meningiomas orbitarios que parecen originarse de las células aracnoideas ectópicas de cualquier tejido meníngeo.

El manejo diagnóstico se ha modificado en los últimos diez años, desarrollándose nuevos métodos diagnósticos de la imagen (Ecografía, TC alta resolución, RMN), técnicas de biopsia con aguja fina (PAAF), biopsia endoscópica. La siguiente aportación ha sido en el campo del tratamiento quirúrgico, con las técnicas de microcirugía intraocular en un intento de preservar el máximo de agudeza y campo visual que ha ido perdiendo relevancia hasta quedar relegada en los últimos años a los pacientes que han perdido totalmente la visión. El tercer pilar de desarrollo, es el papel que ha ido adoptando la Radioterapia como técnica conservadora en sustitución de la cirugía, en especial, el uso ya reconocido de la RTEF como técnica electiva estándar en los pacientes que mantienen un cierto grado de visión.

\section{Material y métodos}

Presentamos como documento gráfico un caso tratado en nuestro servicio con RTEF en el año 2000.

\section{Caso clínico}

Paciente mujer de 52 años, sin antecedentes familiares de interés oncológico y como antecedentes personales había sido intervenida de síndrome de túnel carpiano en la mano izquierda y nódulos mamarios benignos. En el momento del diagnostico estaba en tratamiento con suplementos de calcio y calcitonina por osteoporosis. Desde noviembre de 1999 notaba perdida de visión en ojo izquierdo, junto con dolor ocular ocasional y conjuntivitis bilateral. Vista por oftalmología se apreció en la exploración de fondo de ojo "edema de papila" unilateral a nivel de ojo izquierdo. No se observó alteración en los movimientos oculares de ambas órbitas. Se realizaron las siguientes exploraciones complementarias.

- TC de órbita: en vértice de órbita izquierda y en íntimo contacto con nervio óptico, se visualiza una masa de $1 \mathrm{~cm}$ de diámetro mayor de localización intraconal que captaba contraste de forma in- 


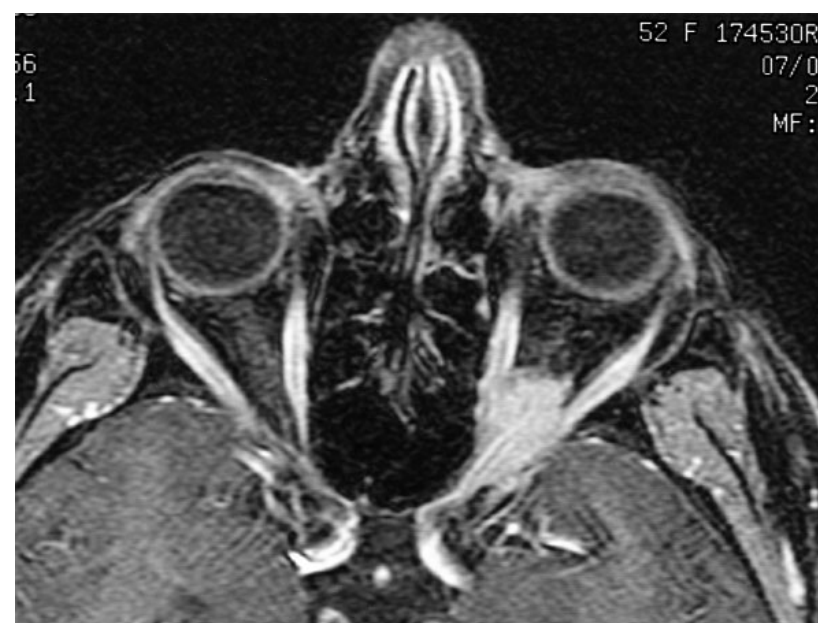

Figura 1. RMN con contraste del MVNO al diagnóstico.

tensa y homogénea, observándose un engrosamiento difuso de la vaina del nervio óptico formando una imagen en "railes de tranvía". No había evidencia de agrandamiento del agujero óptico ni invasión intracraneal; no se aprecian erosiones óseas (Figura 2).

- RMN cerebral y de órbita: masa de señal isointensa con el parénquima cerebral en todas las secuencias que ocupa el agujero óptico izquierdo y que mostraba captación intensa y homogénea de contraste. La lesión englobaba el nervio óptico izquierdo que se encuentra discretamente comprimido. El tamaño aproximado de la lesión era de $2 \times 1 \times 1$ $\mathrm{cm}$. Los hallazgos radiológicos sugerían la presencia de meningioma aunque no se podían descartar otras posibilidades como un tumor de la vaina nerviosa (Figura 1).

- Estudio oftalmológico: mostró en la agudeza visual una pérdida de la visión a 1/10 y el estudio campimétrico mostró un déficit en ojo izquierdo conservando parcialmente la visión en cuadrante superior interno.

Valorado por el Comité de Radiocirugía (RC) se aceptó el caso para RTEF, siendo irradiado con un Acelerador Lineal con fotones de $6 \mathrm{Mv}$, con colimadores cónicos de 10 y $25 \mathrm{~mm}$, con 2 isocentros, administrándose 8 arcos esterotáxicos y una dosis total de 52,2 Gy en fracciones de 1,8 Gy, hasta un total de 29 fracciones. Se eligió como referencia la Isodosis de Cubrimiento del 80\% (Figura 3).

La paciente respondió clínicamente con la desaparición de la sintomatología dolorosa orbitaria y el

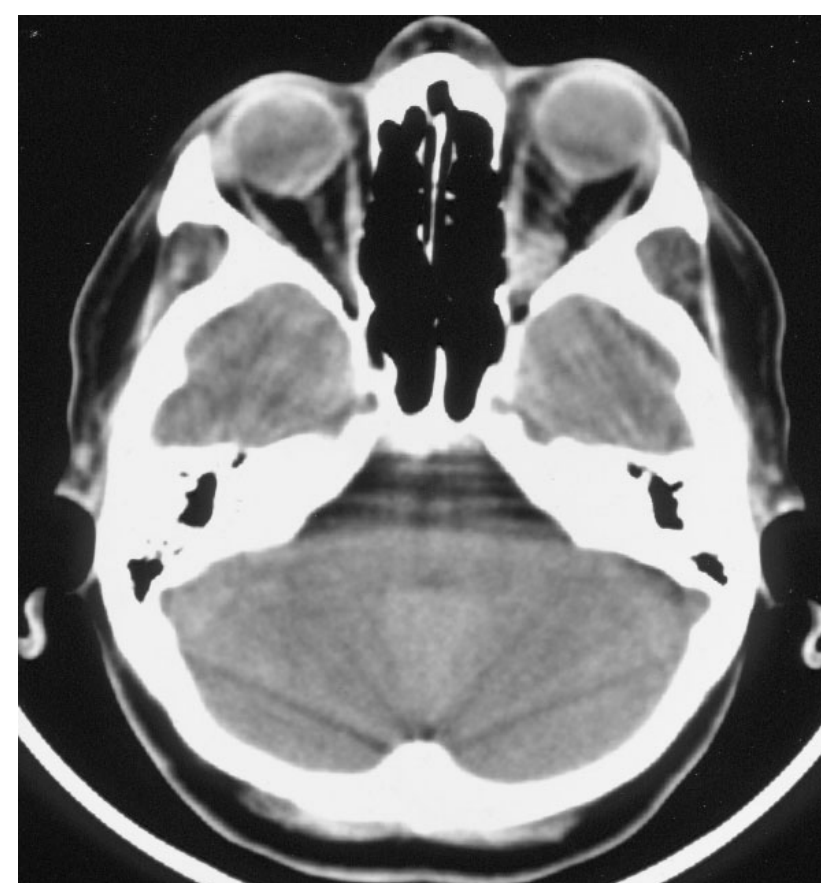

Figura 2. TC de orbita al diagnóstico de MVNO.

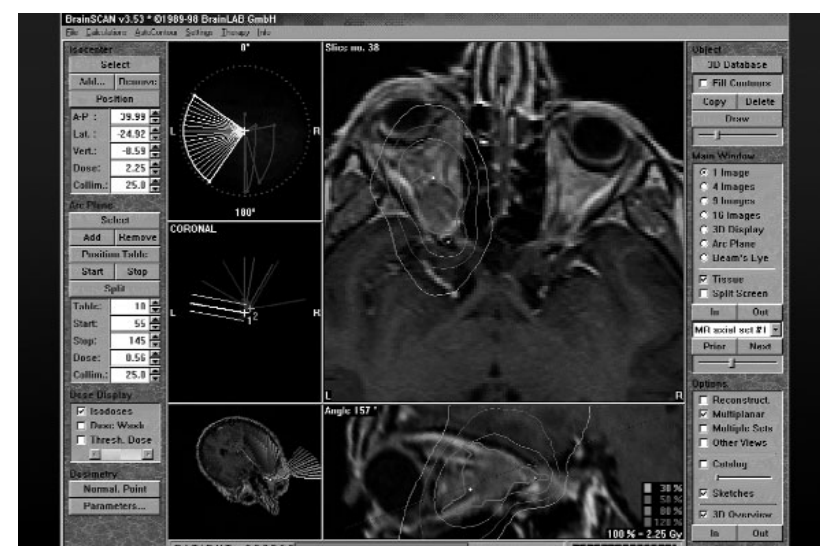

Figura 3. Planificación del tratamiento con RTEF del MVNO en un Acelerador Lineal.

exoftalmos ya en el primer control semestral. Las exploraciones con RMN confirmaron en el primer control a los seis meses una evolución favorable, con una disminución significativa del tamaño tumoral, quedando zonas de captación alrededor del agujero óptico, sugestivas de restos tumorales de meningioma que han permanecido estables en los controles posteriores (Figura 4). Los estudios oftalmológicos demostraron mejora de cierto grado de visión, situación que se ha mantenido con el paso del tiempo. La paciente ha sido etiquetada en remisión 


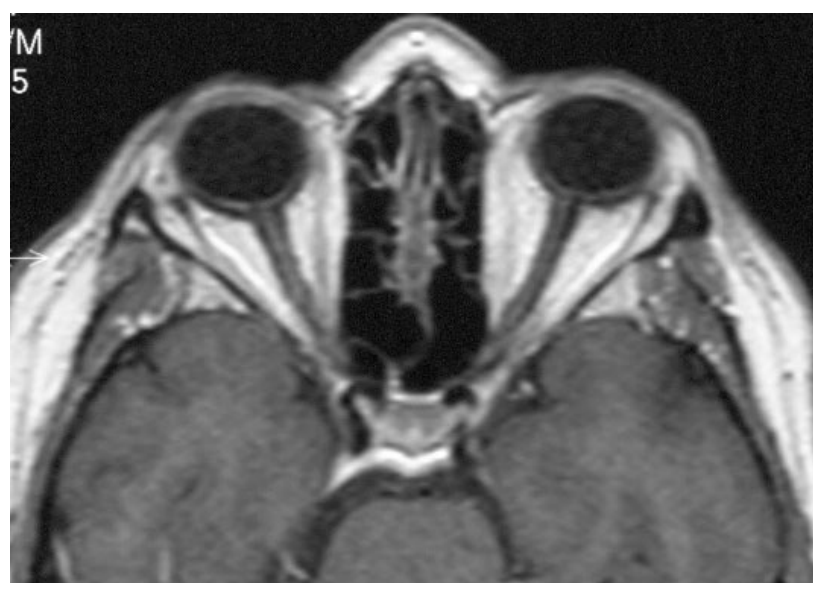

Figura 4. RMN de control a los 18 meses del tratamiento con RTEF.

clínica parcial/completa. No ha presentado signos de toxicidad a nivel de la vía óptica hasta el momento actual.

\section{Discusión}

El MVNO es una entidad rara cuyo tratamiento ha estado sometido a controversia, ya que el mismo incluye la observación, la cirugía y la radioterapia. Con el advenimiento de nuevos métodos de imagen como la RMN/TC de Alta Resolución, se ha podido precisar más el diagnóstico en estadios más precoces, y ha permitido la realización de terapéuticas menos agresivas.

Es necesario conocer de manera precisa el desarrollo embriológico y la anatomía de nervio óptico, para poder entender la fisiopatología de esta rara entidad y su historia natural.

\section{Embriología del nervio óptico}

Para entender la historia natural del MVNO recordaremos que en las etapas incipientes del desarrollo, el cáliz ocular está unido al cerebro por el pedículo óptico que tiene en la superficie ventral, llamada cisura coroidea. Las fibras nerviosas de la retina que envuelven al cerebro, se encuentran entre las células de la pared interna del pedículo. Durante la séptima semana de vida intrauterina, la hendidura ocular fetal se cierra, y se forma un túnel de poco calibre dentro del pedículo óptico. A causa del número constantemente creciente de fibras nerviosas que se dirigen al cerebro, la pared interna del pedículo crece hasta fusionarse con la pared externa. Las células de la capa interna brindan una red de células de neuroglía que dan sostén a las fibras del nervio óptico. El pedículo se convierte en nervio óptico, el centro presenta la arteria hialoidea, que ulteriormente se llamará arteria central de la retina ${ }^{1}$.

\section{Anatomía del nervio óptico}

El nervio óptico es un nervio sensorial que depende del cerebro y representa el pedículo que une la retina al neuroeje. Nace en las células ganglionares de la retina, converge hacia la papila óptica, atraviesa posteriormente la coroides y la esclerótica, constituyendo el ya denominado nervio óptico. Del globo ocular va hacia atrás y adentro, atravesando sucesivamente la cavidad orbitaria y el conducto óptico en la cavidad craneal y termina en el ángulo anteroexterno del quiasma óptico. Las meninges forman alrededor del nervio óptico tres vainas concéntricas (piamadre, aracnoides y duramadre) en todo su trayecto, llegando hasta el mismo globo ocular².

\section{Historia natural}

\section{Origen}

Los MVNO se encuentran localizados dentro de la órbita pudiendo originarse como un crecimiento primario de las meninges del nervio óptico o que pueden invadir la órbita de manera secundaria al lugar de origen dentro de la cavidad craneal. En teoría un meningioma orbitario puede también originarse del tejido meníngeo ectópico, el cual ha emigrado a la órbita durante el periodo embrionario.

Los MVNO se originan en la aracnoides que rodea al nervio óptico, en cualquier parte de su recorrido, pero generalmente en la vecindad del agujero óptico situado en el vértice de la órbita. Estos tumores primarios están localizados dentro del espacio subdural del nervio óptico y, a veces, son difíciles de diferenciar de los meningiomas secundarios.

\section{Curso clínico}

El MVNO crece desde el espacio subdural, comprimiendo la duramadre y el aporte sanguíneo de la piamadre. Esto puede causar atrofia con profunda 
perdida visual, sin invasión propia del nervio. Cuando invade el globo ocular puede producir un desprendimiento de retina. Cuando el tumor sale al interior de la órbita a través de la vaina dural, invade la musculatura extraocular y afecta a otras estructuras perineurales, entonces da lugar a un exoftalmos progresivo, que puede ir acompañado de paresia del globo ocular en función del músculo afecto. El dolor aparece por compresión e invasión de los nervios sensoriales. Cuando hay invasión intracraneal pueden afectarse estructuras como el quiasma óptico, arteria carótida interna, nervio óptico contralateral y silla turca. En casos muy avanzados pueden afectar a estructuras vitales y producir muerte del paciente ${ }^{3}$.

TABLA I

\begin{tabular}{|lc|}
\multicolumn{1}{c}{ Síntomas de Presentación del MVNO } \\
\hline Síntomas & \% Pacientes \\
\hline Pérdida de visión & 77 \\
Visión oscura transitoria & 23 \\
Cefaleas & 9 \\
Proptosis & 9 \\
Estrabismos & 9 \\
Ceguera de abducción & 5 \\
Asintomáticos & 5 \\
\hline
\end{tabular}

\section{Anatomía Patológica}

Los meningiomas del nervio óptico comparten las mismas características histológicas que los meningiomas intracraneles, aunque dos variantes histológicas de meningiomas son características de los MVNO. El tipo histológico meningotelial o tipo sincitial, y el tipo mixto o transicional con o sin cuerpos de psammoma. Las otras variantes de meningiomas denominados fibroblásticos o angioblásticos son encontradas en la órbita por invasión secundaria de esta localización, cuyo origen proviene de la invasión a través de la cavidad intracraneal y dan lugar a los meningiomas secundarios del nervio óptico ${ }^{3}$.

\section{Manejo diagnóstico}

El diagnóstico de MVNO se establece por una clínica sospechosa y signos característicos en explo- raciones de imagen y funcionales ${ }^{5}$. En los últimos años se ha facilitado con el advenimiento de la Tomografía Computerizada (TC) y la Resonancia Magnética Nuclear (RMN). En ellas, las imágenes son típicas, destacando un engrosamiento del nervio óptico en ocasiones acompañado de calcificaciones intratumorales rodeando al nervio óptico. Esto hace posible establecer un diagnóstico definitivo sin biopsia $^{6}$.

- Exploración Oftalmológica: se observa disminución de la agudeza visual, proptosis axial, edema de papila, atrofia óptica, defectos del campo visual con alteraciones que progresan desde agrandamientos de la mancha ciega hasta escotomas centrocecales, defectos altitudinales o constriciones périféricas, trastornos en la motilidad, diplopia y defecto pupilar aferente. Un signo típico es el de HoytSpencer, constituido por la presencia shunts retinociliares sobre el disco óptico, que actúan como "by pass" para mejorar la irrigación del nervio óptico comprometido por el efecto compresivo del meningioma.

- Radiología simple de órbita: aporta pocos signos en estadios precoces. En los estadios avanzados o tardíos se pueden visualizar calcificaciones y cambios de esclerosis o hiperostosis en el canal óptico y/o agrandamiento del mismo ${ }^{22}$.

- Ecografía orbitaria: se observa un ensanchamiento del nervio óptico con una ecogenicidad media (que lo diferencia del glioma del nervio óptico) y con una reflectividad media baja ${ }^{7}$. Adicionalmente se pueden encontrar anomalías en el patrón del nervio, como acentuación o calcificación de las vainas que lo recubren ${ }^{22}$.

- TC de órbita y SNC: ofrece una imagen patente de engrosamiento isointenso o hiperintenso del nervio óptico, que generalmente aumenta con la inyección de contraste. Un signo característico, que aparece cuando se afecta solo la vaina y se respeta el nervio, es la radiotransparencia central o "signo de rail" en el corte axial o del "donut" en el coronal. Este signo nos permite diferenciarlo del glioma del nervio óptico. En ocasiones podemos ver calcificaciones del nervio o hiperostosis en los huesos veci$\operatorname{nos}^{6-8}$. Algunos MVNO pueden asociarse con Pneumosinus Dilatans (expansión de la neumatización del seno) pudiendo verse involucrados el seno esfenoidal, etmoidal o frontal ${ }^{22}$.

- $R M$ de órbita y SNC: es la técnica óptima para 
un diagnóstico preciso y precoz de los meningiomas intracanaliculares y para la estimación del grado de extensión intracraneal de todos estos tumores. En secuencias T1 y T2 se observa una señal isointensa o discretamente hiperintensa respecto al nervio óptico normal. Los estudios en secuencia T1 con refuerzo de gadolinio, permiten observar una hiperintensidad de la zona tumoral, al contrario de lo que sucede con los gliomas del nervio óptico ${ }^{6-8}$.

\section{Manejo terapéutico de los MVNO}

\section{El papel de la Cirugía}

Existe controversia en el manejo de los tumores del nervio óptico. El tratamiento quirúrgico ha planteado problemas desde muy antiguo a diferentes autores clásicos. Byers en 1901, nos habla de la imposibilidad de resecarlos en su totalidad en un único tiempo quirúrgico, debido a la afectación intracraneal que a veces acompaña al tumor. Comentarios en la misma línea se han vertido por autores, como Arnold Knapp, Walter Dandy y Harvey Cushing, que afirman que cuando un tumor orbitario es inextirpable completamente, se puede realizar un abordaje del mismo mediante una craneotomía, aunque las secuelas que deja (ceguera) puedan ser inaceptables ${ }^{9}$.

La cirugía se establece cuando es necesario obtener una muestra de tejido porque existe duda en el diagnóstico diferencial con otras entidades, como la neuritis óptica. El papel de la cirugía depende de la localización del tumor, estado visual y la edad de presentación. Los criterios de cirugía agresiva han sido defendidos por diferentes autores, como Alper, Clark y Wright, en pacientes que tienen tumores localizados posteriormente y asociados a una perdida de la visión progresiva. Está especialmente indicada en pacientes jóvenes, de menos de 40 años de edad, ya que como se ha comentado anteriormente tienen un curso más rápido y agresivo con precoz invasión de la base de cráneo. Los pacientes mayores de 40 años, que tienen inicialmente tumores de localización anterior y de pequeño tamaño, se pueden dejar en observación cada 3 a 6 meses. Si posteriormente progresan, serán sometidos a cirugía descompresiva y/o extirpación del tumor localizado en la órbita, respetando la integridad del nervio óptico ${ }^{9-12}$. En una revisión realizada por Dutton, se produjo recidiva tumoral en un $25 \%$ de los casos tratados median- te extirpación quirúrgica. Cuando la extirpación es incompleta va asociada con una mayor incidencia de extensión hacia el quiasma o de invasión difusa de la órbita, requiriendo la exanteración orbitaria en estos $\operatorname{casos}^{14,27}$.

\section{El papel de la Radioterapia}

El manejo terapéutico de los MVNO ha variado en los últimos años, sobre todo, en base a la experiencia adquirida con el tratamiento radioterápico en los meningiomas intracraneales o de localización intraorbitaria. La incorporación de la radioterapia al patrón de cuidados de MVNO, no ha estado exenta de dificultades dado el elevado porcentaje de complicaciones que presentaba con las técnicas disponibles hasta mediados de los años 70. Es a partir de ese momento, con la generalización de los Aceleradores Lineales, cuando aparecen las primeras series de pacientes tratados con radioterapia.

\section{- Radioterapia Convencional}

Smith JL y otros, publican en 1981 una serie de 5 mujeres diagnosticadas de MVNO que habían sido tratadas desde 1975. Estas pacientes fueron tratadas en el rango de dosis de 4000 rads y con carácter paliativo, obteniéndose mejoría en la agudeza visual, estabilización e incremento en el campo visual, con disminución del tamaño o incluso total regresión de las venas opticociliares.

Kennerdell y otros, publicaron una serie de 38 pacientes, con diferentes actitudes terapéuticas, como fueron la observación en 18 pacientes que presentaban una visión mínima o simplemente el ojo estaba ciego. La radioterapia fue utilizada en 6 pacientes con perdida progresiva del campo de visión y recibiendo aproximadamente 5500 rads entre 28 y 32 fracciones. La mejora de la visión se mantuvo entre 30 y 84 meses. Se combinó la cirugía y la radioterapia en tres pacientes en los que la cirugía no fue completa. La mejoría de la misma se mantiene entre 6 y 9 años de seguimiento. Otros dos pacientes con excisión subtotal no fueron tratados inicialmente, pero si fueron tratados con posterioridad cuando las lesiones progresaron, con empeoramiento de la agudeza visual ó perdida de la visión de los colores. Tras tratamiento con RT con dosis de 5500 rads, el campo visual se mantuvo en estos pacientes entre 3 y 6 años. Otro hecho destacable es la ausen- 
cia, de complicaciones derivadas del tratamiento durante todo el periodo de seguimiento.

\section{- Radioterapia Conformacional Tridimensional (RTC-3D)}

Lee y otros, publican la experiencia con un paciente varón de 43 años. Fue tratado con Radioterapia Conformacional Tridimensional (RTC3D), recibiendo una dosis total de 5040 cGy administradas a 180 cGy/día, durante seis semanas. Una semana después de finalizar el tratamiento, el paciente había mejorado de manera significativa de su función visual ${ }^{15}$.

Moyer y otros publicaron los resultados de una mujer de 35 años aquejada de un MVNO. Fue tratada con RTC3D alcanzando una dosis total de 5040 cGy en 42 días, mediante la administración de seis haces conformados mediante bloques. Los resultados objetivaron una mejoría en la agudeza visual a $\operatorname{los} 4,8$, y 24 meses después del tratamiento ${ }^{16}$.

Otra revisión bibliográfica llevada a cabo por Melian y Jay les lleva a la conclusión final de que la RT disminuye o retrasa el crecimiento tumoral para la mayoría de los pacientes y mejora la visión en algunos ${ }^{25}$.

Por último, Carrasco y Penne realizaron una revisión de los estudios publicados en los dos últimos años, de un total de 62 pacientes tratados con RT fraccionada. El rango de dosis osciló entre los 4000 a 5600 cGy. Las técnicas utilizadas fueron con RTC-3D y la mediana de seguimiento estaba comprendida entre los 22 y 51 meses. Los resultados obtenidos fueron que en un 40,3\% de los casos, la agudeza visual se mantuvo estable, en un $41,2 \%$ había mejorado su visión y un 18,4\% había empeorado su agudeza visual. Como conclusiones establece que la RTC-3D es una modalidad terapéutica que se va consolidando como tratamiento electivo en el MVNO consiguiéndose un bloqueo en la progresión tumoral acompañada con una mejoría o estabilización de la agudeza visual ${ }^{26}$.

\section{- Radioterapia Estereotáxica Fraccionada (RTEF)}

Existe escasa referencia en la literatura de la RETEF en los MVNO. A pesar de que en los últimos cuatro años están apareciendo publicaciones de series de casos, en el momento actual es dificulatoso establecer recomendaciones con un nivel de evidencia suficiente.
Liu y colaboradores, publicaron una serie de 5 casos que presentaban deterioro de la visión con disminución del campo visual, agudeza visual y alteración del color. Todos fueron tratados con dosis total de 45 a 54 Gy, a 1,8 Gy por fracción, en 25 a 30 fracciones. El seguimiento se realizó entre 1 y 7 años, no hallándose cambios en el tamaño de la lesión de las imágenes de resonancia magnética. Pero cuatro pacientes experimentaron mejoría en la agudeza visual, campo visual y visión del color dentro de los 3 meses posteriores a la administración de la RTEF. Uno de los pacientes permanece estable, sin evidencia de deterioro visual o enfermedad progresiva. No hubo neuropatía óptica inducida por radiación ${ }^{17}$.

Otra serie importante de tratamiento con RTEF en los MVNO, es la publicada por Becker y colaboradores. En ella se trataron 39 pacientes (15 primarios y 24 secundarios). La dosis total prescrita fue de 54 Gy, en 28 fracciones dentro de las 5,5 semanas, usando un acelerador lineal con un rango de energía de $6 \mathrm{Mv}$. Todos los pacientes experimentaron estabilización de la enfermedad. En el momento del análisis, la totalidad de los pacientes están vivos sin evidencia de progresión de la enfermedad. No hubo que lamentar toxicidad crónica a nivel del nervio óptico afecto ${ }^{18}$.

Kerty, publica un caso como experiencia propia y realiza una puesta al día del manejo de los MVNO y los compara con la amplia experiencia de la observación y la cirugía en esta entidad. El autor llega a la conclusión de que la RDCEF puede conseguir la estabilización ó mejoría de la función visual en los $\mathrm{MVNO}^{22}$.

Una revisión publicada por Baumert y otros, estudió a 23 pacientes en observación, que progresaron a nivel del tumor de manera fehaciente. Todos los pacientes recibieron RTEF, con una mediana de dosis total de 50,4 Gy en 6 semanas. Tras un seguimiento mediano de 20 meses (rango 1-68 meses) en un $95 \%$ de los casos (21 de 22) fue controlada su función visual. Un total de 16 pacientes tuvieron mejoría visual y en otros 5 pacientes la misma permaneció estable. Solamente empeoró la visión en un paciente. En 6 pacientes hubo mejoría del dolor y en un paciente mejoró también la proptosis. Solo cabe destacar como toxicidad relevante un paciente presentó un cuadro de retinitis rádica y hemorragia vítrea que fue vista a los 4 años después del procedimiento ${ }^{24}$. 
En el año 2004 Melian y Jay revisaron el papel de la RTEF en el MVNO y comentan dos series publicadas en el año 2002. Una de ellas fue publicada por Pitz y colaboradores en 15 pacientes que habían progresado previamente al tratamiento con RT. La dosis total administrada fue de 54 Gy en 30 fracciones. En una primera fase recibieron 50,4 Gy en la lesión, mas un margen de $5 \mathrm{~mm}$., y en una segunda fase 3,6 Gy a la lesión más un margen de $2 \mathrm{~mm}$. Con un seguimiento medio de 37 meses, 7 pacientes tenían mejoría de la función visual y en ninguno de los otros pacientes hubo empeoramiento de la misma. Otra serie analizada fue la de Andrews y colaboradores, que publicaron la experiencia Willis Eye Hospital en 30 pacientes con MVNO. Se utilizó un acelerador lineal provisto de colimadores cilíndricos. La mediana de dosis total fue de 51 Gy. Un $42 \%$ de los pacientes presentaban mejoría de la visión y un $50 \%$ visión estable con una mediana de seguimiento de 22 meses $^{25}$.

Por último Kwon y otros, presentaron una serie de 3 pacientes (2 gliomas del nervio óptico y un MVNO) tratados con radiocirugía con gamma kniffe y con un rango de dosis entre los 8 y $15 \mathrm{~Gy}$. La agudeza visual mejoró en los tres pacientes y el tamaño tumoral disminuyó progresivamente a lo largo del seguimiento con RM. Se observó resolución del exoftalmos en 1 paciente. Los autores de este artículo recomiendan utilizar dosis de $8 \mathrm{~Gy}$, cuando hay conservación de la visión normal y dosis más altas cuando hay deterioro completo de la misma, entrando en contradicción con las recomendaciones mayoritariamente aceptadas ${ }^{27}$.

\section{Conclusiones}

El MVN es una entidad cuyo manejo terapéutico se ha modificado de manera significativa, ocupando un lugar relevante la RT en sus diferentes formas de administración. La RTEF ha conseguido estabilización de la enfermedad en prácticamente, la totalidad de los pacientes. En un porcentaje muy alto la sintomatología ha mejorado de manera subjetiva y objetiva (test de función visual, y hallazgos en RMN/ TC de Alta Resolución), quedando como técnica de elección en los pacientes con pérdida de función visual.

Otro aspecto relevante, tanto en las series publi- cadas como en nuestro caso, es la ausencia de toxicidad crónica a nivel de la vía óptica. Esto es especialmente evidente cuando administramos dosis totales por debajo de los $54 \mathrm{~Gy}$, con un fraccionamiento convencional que no supere los incrementos de dosis diarios de $2 \mathrm{~Gy}^{19-21,23}$.

En el caso de RC de sesión única, las dosis utilizadas oscilarán entre los 10 y 14 Gy., en función de la isodosis de referencia (80-100\%) que se elija. Esta técnica solo estará indicada en aquellos pacientes con pérdida de visión completa, ya que las dosis necesarias para el control a largo plazo, están por encima de los niveles de tolerancia de la vía óptica que es de 8 Gy.

La cirugía solo estará indicada en casos de pacientes con enfermedad rápidamente progresiva acompañados de pérdida total de la visión con carácter irreversible, en los que resulta imprescindible eliminar un compromiso mecánico secundario al gran tamaño tumoral, pudiéndose en estos casos combinarse con la RTEF o la RC.

Para finalizar diremos que la observación o vigilancia quedará reservada a aquellos pacientes mayores, con escasa sintomatología y que no presentan deterioro de la función visual.

Como recomendación final y dada la escasa experiencia acumulada hasta la actualidad tanto a nivel nacional como incluso a nivel internacional, sería necesario plantearse un grupo de trabajo de carácter multidisciplinar (Oftalmólogos, Neurocirujanos, Oncólogos Radioterápicos, Diagnóstico por Imagen) con el objetivo de compartir experiencias, desarrollar guías de práctica clínica y abrir nuevas líneas de investigación clínica.
Correspondencia:

Dr. M. Caeiro Muñoz

O Fuxon, 14

E-36958 Tirán-Moaña (Pontevedra) caeiro.munoz@cmpont.es 


\section{Bibliografía}

1. Embriología humana (III edición), Jan Langman, Capítulo 16, pag 339-340-

2. Anatomía humana (descriptiva, topogáfica y funcional) Tomo I, Nervios de la Cabeza y del Cuello, pag 239-241.

3. Melvin G Alper. Management of Primary Optic Nerve Meningiomas, Currente Status - Therapy in Controversy, J Clin Neuro-ophtalmol. 1 101-117, 1981.

4. Patrick A Sibony, Howard T. Krauss, J S Kennerdell, J C Maroon: Optic Nerve Sheath Meningiomas. Clinical Manifestations. Ophthalmology 91: 1313-1326, 1984.

5. J Michel Henk: Tumors of the orbit, Oxford Textbook of Oncology, vol (1),Edited by Michael Peckham, Herbert M Pinedo, and Umberto Veronesi, pag 944-953.1995.

6. Gerd Becker, Branislav Jeremic, Susanne Pizt, Markus Buchgeister et al: Stereotactic Fractionated Radiotherapy in patients with Optic Nerve Sheath Meningioma, Int J Radiation Oncology Biol Phys, Vol 54, No. 5 pp1422-1429, 2002.

7. Publicaciones Nexus Ediciones, S.L. Meningioma primario de la vaina del nervio óptico: diagnostico, estudio anatomopatológico y tratamiento, Disponible en: http// www.nexusediciones.com.

8. Arruga J, Prat J, Díaz MM. Gliomas ópticos benignos y meningiomas del nervio óptico. Publicaciones Nexus Ediciones SL, Disponible en: http//www.nexusediciones.com.

9. MG Alper, Management of Primary Optic Nerve Meningiomas, Current Status-Therapy in Controversy, J Clin. Neuro-ophtalmol. 1: 101-117, 1981.

10. WC Clark, CS Theofilos, JC Fleming. Primary optic nerve sheath meningiomas, Report of nine cases. J Neurosurg 70: 37-40, 1989.

11. JE Wright, AA Mcnab, I Macdonald. Primary optic sheath meningioma. British Journal of Ophtalmology, 1989, 73, 960-966.

12. Gabib A, G Sabibov, Samuil M, Blinkob, Vasiliv et al. The mamagement of optic nerve meningiomas and gliomas. J Neurosurgery 68: 889-893, 1988.

13. Smith JL,Vuksanovic MM, Yates BM, Bienfang DC. Radiation Therapy for Primary Optic Nerve Meningiomas. J Clin. Neuro-ophthalmol. 1: 85-99, 1981.

14. Kennerdell S, Maaron JC, Malton, Warren FA. The Management of Nerve Sheath Meningiomas. Am J Ophthalmol. 106: 450-457, 1988.

15. Lee AG, Woo Y, Miller NR, Safran AB, Grant WH, Improvement in Visual Function in an Eye with a Presumed Optic Nerve Sheath Meningioma after Treatment with Tree-Dimensional Conformal Radiation therapy. Journal of Neuro-Ophtalmology 16(4): 247-251,1996.
16. Moyer PD, Golnick KC, Breneman J. Treatment of Optic Nerve Sheath Meningioma With Three-Dimensional Conformal Radiation Am J Ophthalmol 2000; 129: 694-696.

17. Liu JK, Forman S, Hershewe, GL, Moorthy CR, et al. Optic Nerve Sheath Meningiomas: Visual Improvement after Stereotactic Radiotherapy. Neurosurgery 50: 950-957, 2002.

18. Becker G, Jeremic B, Pitz S, Bucheister M, et al. Stereotactic Fractionated Radiotherapy in patients with optic nerve sheath meningioma. Int J Radiation Oncology Biol Phys2002;54(5):1422-1429.

19. Maguire PD, Clough R, Friedman H, Halperin et al. Fractionated External Beam Radiation Therapy for meningiomas of the cavernosus sinus. Int. J Radiation Oncology Biol Phys 1999, 44 (1), 75-79.

20. Tsihler RB, Loffleler JS, Lunsford LD, Duma C, et al. Tolerance of cranial nerves of the cavernous sinus to radiosurgery. In J Radiation Oncology Biol Phys 1999, 27, 215 221.

21. Parsons JT, Bova FJ, Fitzgerald CR, Mendenhall W M, et al. Radiation optic neuropathy after Megavoltage External - Beam Irradiation: Analisys of Time-Dose Factors. Int. Radiation Oncology Biol. Phys. 1994, 30 (4), 755-763.

22. Kertey E. Optic nerve sheath meningioma-from expectation to active treatment. Tidsskr Nor Laegeforen. 2005 Feb 17; 125(4):429-30 (Abstract, Artículo en Noruego).

23. Pérez Moreiras J, García Allut A, Prada Sánchez C, Coloma Becaos J, Pérez Becerra E. Meningiomas del Nervio Optico. Capítulo 12, páginas 173-191. Meningiomas 2002, Editado por Manuela Lema Bouzas y Miguel Gelabert Gonzalez patrocinado por Instituto Universitario de las Ciencias Neurológicas "Pedro Barriere de la Maza, promovido por la Universidad de Santiago de Compostela.

24. Baumert BG, Villa S, Studer G, Mirimanoff RO, et al. Early improvements in vision after fractionated stereotactic radiotherapy for primary optic nerve sheath meningioma. Radiother Oncol. 2004 72(2): 169-74.

25. Melian E, Jay WM. Primary radiotherapy for optic nerve sheath meningioma. Seminars in Ophthalmology 2004, 19, (3-4) pp. 130-140.

26. Carrasco JR, Penne RB. Optic nerve sheath meningiomas and advanced treatment options. Curr Opin Ophthalmol 15: 406-410.

27. Kwon Y, Bae JS, Kim JM, Lee do H, y otros. Visual changes alter Gamma Knife Surgery for optic nerve tumors. J Neurosurg (Suppl) 102: 143-146, 2005.

28. Dutton JJ. Optic Nerve Sheath Meningiomas. Major Review. Surv Ophtalmol 37: 167-183, 1992. 